\title{
Nanoscopic Tip Sensors Fabricated by Gas Phase Etching of Optical Glass Fibers
}

\author{
Jörg BIERLICH ${ }^{1 *}$, Jens KOBELKE ${ }^{1}$, David BRAND ${ }^{2}$, \\ Konstantin KIRSCH ${ }^{1}$, Jan DELLITH ${ }^{1}$, and Hartmut BARTELT ${ }^{1}$ \\ ${ }^{1}$ IPHT, Institute of Photonic Technology, Albert-Einstein-Str. 9, 07745 Jena, Germany \\ ${ }^{2}$ EAH, University of Applied Sciences, Carl-Zeiss-Promenade 2, 07745 Jena, Germany \\ ${ }^{*}$ Corresponding author: Jörg BIERLICH $\quad$ E-mail: joerg.bierlich@ipht-jena.de
}

\begin{abstract}
Silica-based fiber tips are used in a variety of spectroscopic, micro- or nano-scopic optical sensor applications and photonic micro-devices. The miniaturization of optical sensor systems and the technical implementation using optical fibers can provide new sensor designs with improved properties and functionality for new applications. The selective-etching of specifically doped silica fibers is a promising method in order to form complex photonic micro structures at the end or within fibers such as tips and cavities in various shapes useful for the all-fiber sensor and imaging applications. In the present study, we investigated the preparation of geometrically predefined, nanoscaled fiber tips by taking advantage of the dopant concentration profiles of highly doped step-index fibers. For this purpose, a gas phase etching process using hydrofluoric acid (HF) vapor was applied. The shaping of the fiber tips was based on very different etching rates as a result of the doping characteristics of specific optical fibers. Technological studies on the influence of the etching gas atmosphere on the temporal tip shaping and the final geometry were performed using undoped and doped silica fibers. The influence of the doping characteristics was investigated in phosphorus-, germanium-, fluorine- and boron-doped glass fibers. Narrow exposed as well as protected internal fiber tips in various shapes and tip radiuses down to less than $15 \mathrm{~nm}$ were achieved and characterized geometrically and topologically. For investigations into surface plasmon resonance effects, the fiber tips were coated with nanometer-sized silver layers by means of vapour deposition and finally subjected to an annealing treatment.
\end{abstract}

Keywords: Optical fiber tips, nanoprobes, gas phase etching, doping, tip shaping, metal coating

Citation: Jörg BIERLICH, Jens KOBELKE, David BRAND, Konstantin KIRSCH, Jan DELLITH, and Hartmut BARTELT, "Nanoscopic Tip Sensors Fabricated by Gas Phase Etching of Optical Glass Fibers," Photonic Sensors, DOI: 10.1007/s13320-012-0085-0.

\section{Introduction}

Silica-based, metal-coated as well as uncoated fiber tips (tapers) are used in a variety of spectroscopic, micro- or nano-scopic sensor applications (e.g. AFM, SNOM, TERS) [1-6] as well in photonic micro-devices [7-14]. The optical resolution e.g. in the case of a scanning probe microscopy system depends on the tip geometry and the diameter of the tapered fiber end, which requires only a few tens of nanometers [15]. In addition to taper drawing or stretching processes [16], the tip shaping of the multimode glass fibers is done primarily by wet-chemical etching usually in the hydrofluoric acid solution [17-20]. Disadvantages of the wet etching process are possible precipitation

Received: 17 July 2012 / Revised version: 31 July 2012

(C) The Author(s) 2012. This article is published with open access at Springerlink.com 
of fluorinated residual components, glass contaminations by condensed reactants and consequently etching inhomogeneity as well as a high surface roughness.

The aim of this study was to investigate the possibility of preparing geometrically predefined, nanoscaled glass fiber tips using a gas phase etching process [21]. The advantage of this approach is that all reaction products are obtained in a gaseous form, and therefore surface condensation can be avoided which often leads to etching inhomogeneity. A further innovation is the utilization of dopant concentration effects [22] on the etching rate of highly doped step-index fibers having a distinct central concentration-dip. The potential for the direct formation of photonic micro-devices at the end or within optical fibers through a selective etching process has been demonstrated for several all-fiber applications such as miniature pressure sensors [9], high-sensitivity Fabry-Perot strainsensors [10-12], inline microcells [13], and openpath micro-resonators [14].

\section{Experimental details}

Technological investigations into the influence of the etching gas atmosphere on the temporal development of the geometrical tip shape and the final geometry were performed at first using undoped silica fibers. At the beginning, a several meters long glass fiber was prepared by fiber drawing, processing a silica preform (F300) into a continuous fiber with a diameter of $125 \mu \mathrm{m}$. Then, the glass fiber was cleaved into $10-\mathrm{cm}$-length samples and positioned in the gas phase $2 \mathrm{~mm}$ above the fluid level of the $40 \%$ hydrofluoric acid (HF) solution at $22{ }^{\circ} \mathrm{C}$. The experimental setup in Fig. 1 shows a HF-vessel, which could be sealed, and an array of typically five fiber samples to be etched. The reaction vessel was separated from the environment by an adjustable gas orifice to apply different HF partial pressures in the etching gas atmospheres. By means of a gas extraction system, the reaction chamber could be deaerated additionally. After a specific reaction time, the fiber samples were removed, and the progress of glass tip shaping was observed by microscopic characterization.

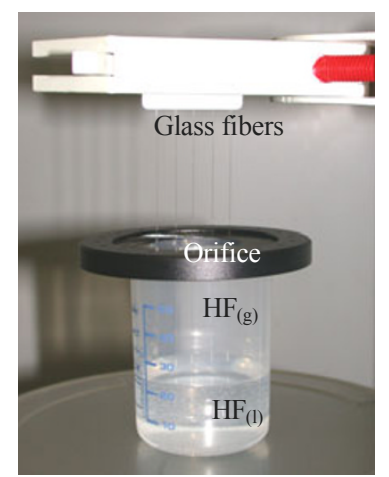

Fig. 1 Experimental etching setup consisting of a closable HF-acid vessel and a fiber sample holder.

The influence of the doping characteristics on the geometrical tip shape was then investigated in phosphorus-, germanium-, fluorine- and boron-doped glass fibers. The fiber samples were drawn from doped MCVD-preforms varying in their doping profile and dopant concentration. Because of the relatively large silica jacket with a lower etching rate than that in the doped central parts of the fiber, the fiber ends were first sealed with a cured acrylate drop [Fig.2(a)], and then the undoped silica jacket was removed without etching of the doped fiber core [Fig. 2(b)]. After this pre-etching step, the acrylate drop was removed, and in a following etching step, the remaining doped core was etched to shape the fiber tip. The HF gas reacted with the doped and undoped silica fibers by reaction with silicon dioxide as well the dopants to form gaseous fluoride compounds. The following chemical equations reflect the reactions of the proceeded gas phase etching processes:

$$
\begin{aligned}
& \mathrm{SiO}_{2}+4 \mathrm{HF}(\mathrm{g}) \rightarrow \mathrm{SiF}_{4}(\mathrm{~g})+2 \mathrm{H}_{2} \mathrm{O} \\
& \mathrm{P}_{2} \mathrm{O}_{5}+6 \mathrm{HF}(\mathrm{g}) \rightarrow 2 \mathrm{POF}_{3}(\mathrm{~g})+3 \mathrm{H}_{2} \mathrm{O} \\
& \mathrm{GeO}_{2}+4 \mathrm{HF}(\mathrm{g}) \rightarrow \mathrm{GeF}_{4}(\mathrm{~g})+2 \mathrm{H}_{2} \mathrm{O} \\
& \mathrm{B}_{2} \mathrm{O}_{3}+6 \mathrm{HF}(\mathrm{g}) \rightarrow 2 \mathrm{BF}_{3}(\mathrm{~g})+3 \mathrm{H}_{2} \mathrm{O}
\end{aligned}
$$

For investigations into surface plasmon resonance effects, the etched fiber tips were vapour-coated with nanometer-sized silver layers 
$[23,24]$. Finally, the metal coated fiber tips were subjected to a high temperature annealing process in order to structure the metal layers.

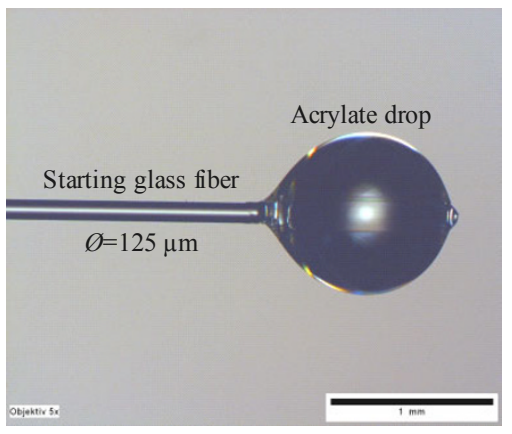

(a)

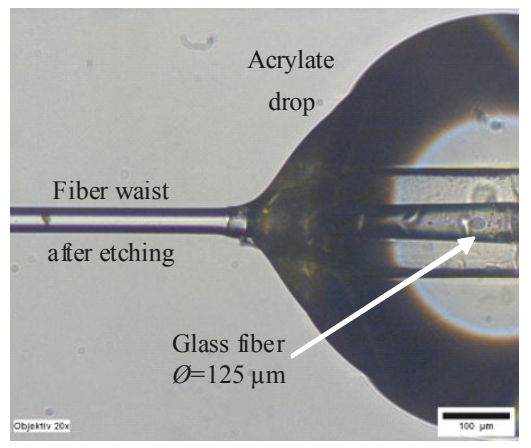

(b)

Fig. 2 Stages of the pre-etching process: (a) before starting the etching treatment for doped fiber structures, the fiber end was sealed with a cured acrylate drop, and (b) after removing of the silica jacket, the doped fiber core remains behind the acrylate drop.

\section{Influence on the etching kinetics}

The influence of the etching gas atmosphere on the tip shaping was investigated at first using undoped silica fibers. The diagrams in Fig. 3 illustrate the dependency of the gas orifice setting on the temporal tip shaping and on the final geometry.

Different shapes of fiber shafts and tip regions were obtained by changing the gas orifice [Fig.3(a)]. By enlarging the orifice size from a small circular hole opening to a slit opening and finally to a full opening, the formation of the axial fiber shaft geometry could be influenced. The diameter of the fiber shaft varied significantly, and the etching time $\left(t_{e}\right)$ to form a fiber tip covered a range from $3 \mathrm{~h}$ to $7 \mathrm{~h}$.
An additional deaeration of the etching gas in the fully opened setting led to a large fiber shaft diameter close to the starting diameter and to a drastic increase in the minimum time for the etching treatment $(12 \mathrm{~h})$. Despite of the gas phase application, a growing damage of the fiber surface was observed with an increasing process time. This led to a degradation of the mechanical stability of the already fragile fiber tips.

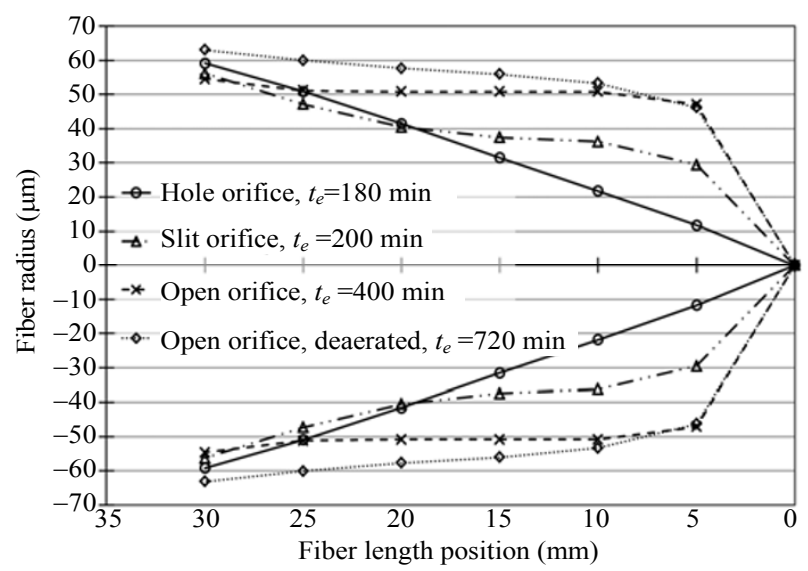

(a)

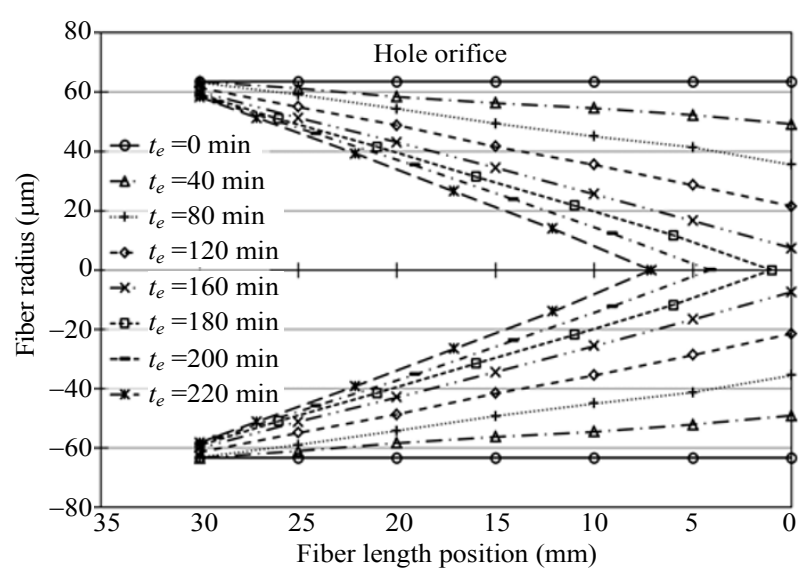

(b)

Fig. 3 Temporal development of the fiber tip shaping: (a) various fiber tips shapes are achieved depending on the etching conditions - the process time $\left(t_{e}\right)$ to shape a fiber tip varies significantly from $3 \mathrm{~h}$ to $12 \mathrm{~h}$ and (b) temporal tip shaping for a narrow hole gas orifice - the time-dependent fiber tapering was found to be very uniform and reproducible.

As an example, Fig. 3(b) shows the temporal development of the tip shape in the case of a narrow hole gas orifice (hole size: $\varnothing=2 \mathrm{~mm}$ ). The formation 
of the fiber tip was found to be very uniform and reproducible. The tapering of the glass fiber end was achieved over a fiber length of several centimeters at different etching rates (Fig. 4). A sharp tip geometry was obtained after a reaction time of $180 \mathrm{~min}$. A continuation of the etching treatment led to the shortening of the tip length and thus to an increase in the fiber tip angle. Tip angles in the range of $0.05^{\circ}$ to $5^{\circ}$ were achieved and could reproducibly be adjusted by enlarging the gas orifice. Independent of the gas orifice setting, the etching rate had a maximum at the origin of the fiber tip and decreases along the fiber length depending on the opening shape of the orifice as well as on the deaeration of the etching gas atmosphere (Fig. 4).

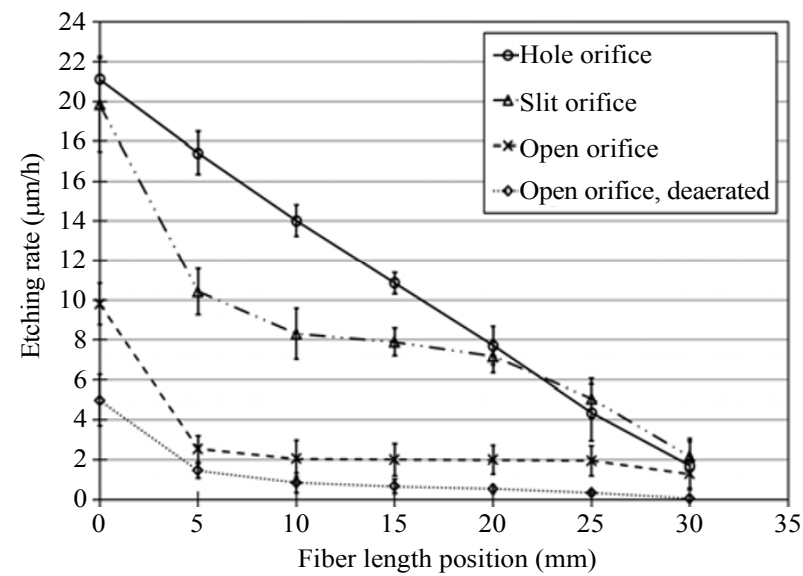

Fig. 4 Different etching rates along the axial fiber position depending on the gas orifice setting.

As an example, Fig. 5 shows a scanning electron microscope (SEM) picture of a resulting fiber tip prepared with slit orifice setting (slit size: $7 \mathrm{~mm} \times$ $28 \mathrm{~mm}$ ). Narrow tips with diameters down to $25 \mathrm{~nm}$ were achieved with pure silica fibers under all etching conditions. However, the tip angles were extremely small even in the case of the obtained maximum value of $5^{\circ}$. Furthermore, the length of the strongly tapered tip region was shown to be in the range of a few millimeters up to $30 \mathrm{~mm}$ depending on the etching gas atmosphere. These geometrical conditions generated rather fragile fiber tips and required careful operation.

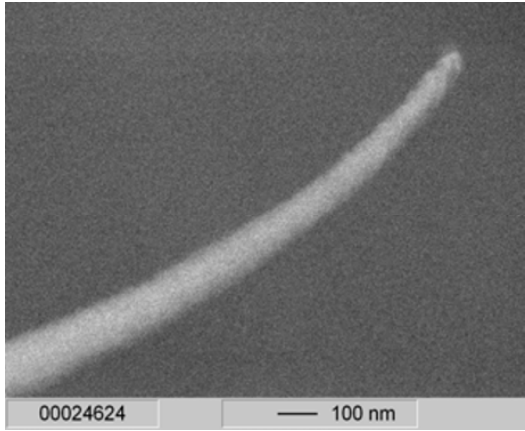

Fig. 5 SEM image of a narrow glass tip etched from a pure silica fiber: tip diameters down to $25 \mathrm{~nm}$ were obtained with a smooth surface quality.

\section{Influence of doping effects}

Next, the influence of the doping effects was investigated in phosphorus-, germanium-, fluorineand boron-doped glass fibers. The fibers were drawn from doped modified chemical vapor deposition (MCVD) preforms which differed in the doping profile and dopant concentration. As an example, Fig. 6 shows the refractive index profile and the associated doping characteristics of a $\mathrm{P}_{2} \mathrm{O}_{5}$-doped step-index preform as well as the cross section micrograph of the resulting glass fiber. The preform was prepared with a highly phosphorus-doped silica core and a lower doped cladding barrier layer. The doped silica core showed an undoped central dip due to the finial preform collapsing step during manufacturing. The doped fiber layers were surrounded by a large-scale silica jacketing. The etching treatment was performed in two process steps. Firstly, the acrylate drop sealed fiber samples were etched to remove the thick-walled undoped silica jacket. In the second step, the remaining doped fiber core was etched to taper the fiber end.

The geometric shaping of the fiber tips is based on the local etching rates of the doped glass structures. Here, the etching behavior correlates with the doping-dependent refractive index profile of the fibers. Pure silica glass shows a comparatively low etching rate. Therefore, strong etching gradients take effect especially in the region of the central index dip, since the dopant concentration is reduced to zero and thus the etching rate is minimized. 


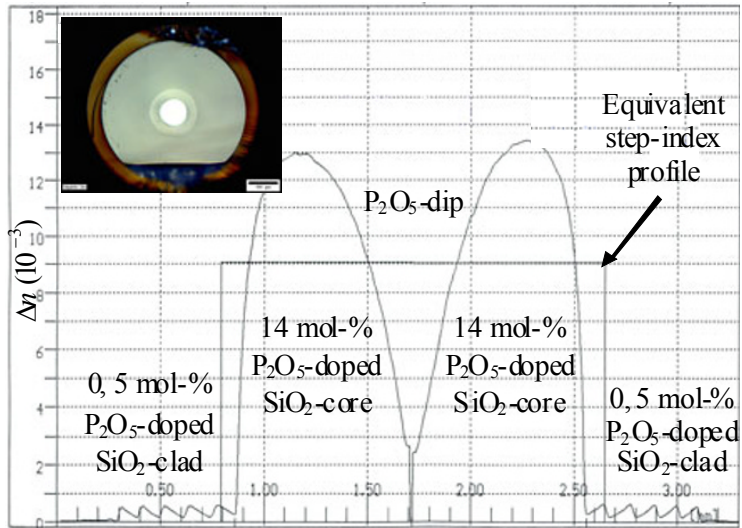

Fig. 6 Radial step-index profile of a phosphorus-doped glass prefrom showing the typical central index dip: the inset shows the cross section of the resulting glass fiber sample.

Figure 7 illustrates the influence of the tested fiber dopants on the etching rate in comparison to an undoped glass fiber (F300) as well as the obtained fiber tip diameters.

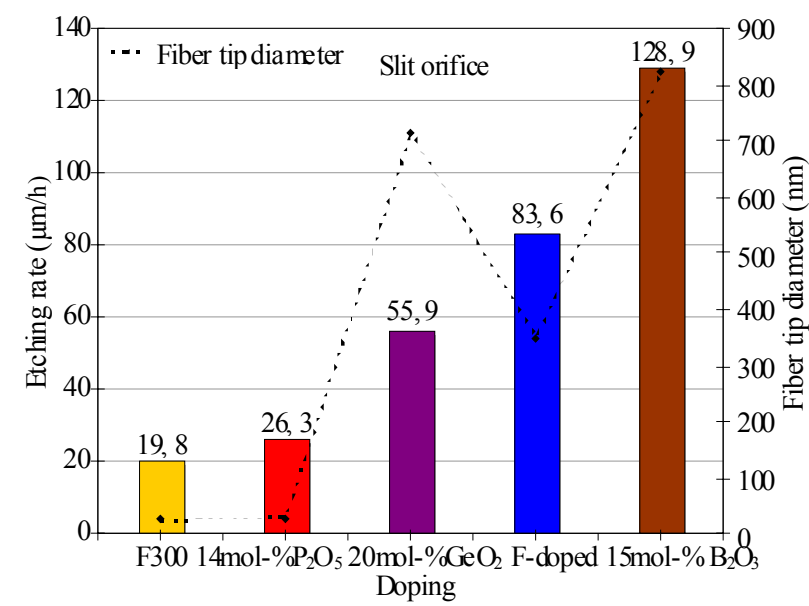

Fig. 7 Influence of different fiber core dopants on the etching rate in comparison to an undoped glass fiber (F300) and the achieved fiber tip diameters.

The pure silica fiber shows the lowest etching rate whereas the tested dopants increased the etching rate significantly by up to a factor of 6 in the case of the boron doping. The achieved fiber tip diameters, from $30 \mathrm{~nm}$ up to $800 \mathrm{~nm}$, and the tip angles tended to decrease with a lower etching rate.

Depending on the doping profile, fiber tips in different shapes were produced. As an example, Figs. 8 and 9 show the resulting tips of phosphorus- and fluorine-doped fiber samples.

The tip formation in the case of the $\mathrm{P}_{2} \mathrm{O}_{5}$-doped sample could be described as an inversion of the refractive index profile (Fig. 6). The central dip of undoped silica produced a sharp glass tip [Fig. 8(a)]. Compared to fiber tips produced by undoped silica fibers, the $\mathrm{P}_{2} \mathrm{O}_{5}$-doped samples showed a distinct transition between the fiber shaft and tip region. The length of the tip regions was significantly lower and appeared to be in the range from $50 \mu \mathrm{m}$ to $150 \mu \mathrm{m}$. This tip geometry led to a higher mechanical stability than that of the pure silica fiber types. The smallest tip radius of about $15 \mathrm{~nm}$ was obtained with the phosphorous-doped glass fibers. In the case of the fluorine-doped fibers, shorter and more obtuse tips were achieved [Fig. 8(b)], however with diameters only above $350 \mathrm{~nm}$ (Fig. 7).

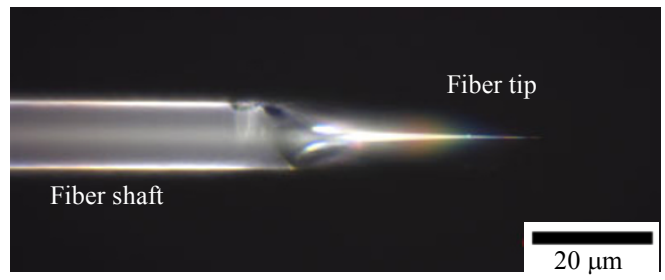

(a)

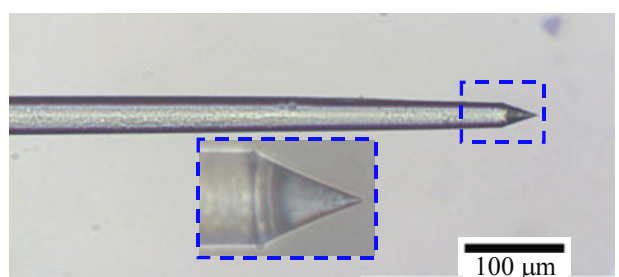

(b)

Fig. 8 Optical micrographs of glass tips etched from a $\mathrm{P}_{2} \mathrm{O}_{5}$-doped (a) and from an F-doped (b) fiber sample.

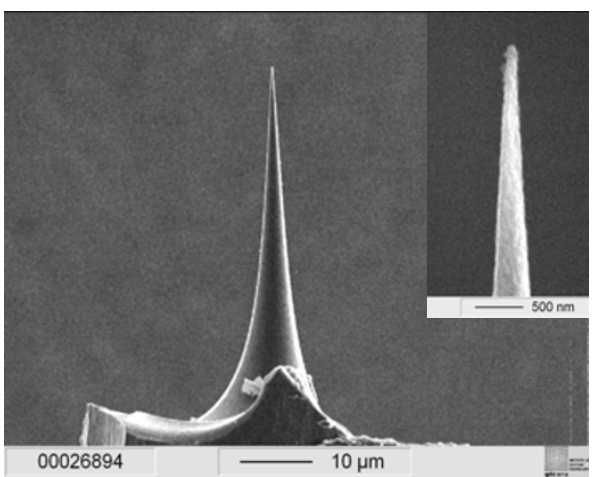

Fig. 9 SEM images along the full tip length and for the tip position (inset) of a $\mathrm{P}_{2} \mathrm{O}_{5}$-based tip.

A very specific fiber tip formation (Fig. 10) was 
achieved in the case of not applying the pre-etching step (see Section 2).

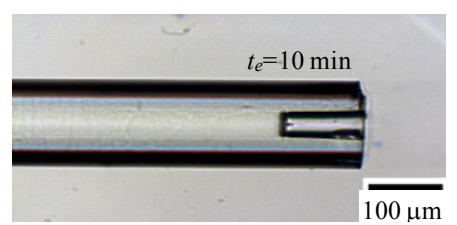

(a)

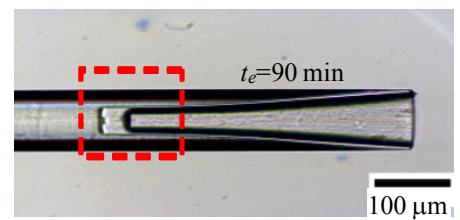

(b)

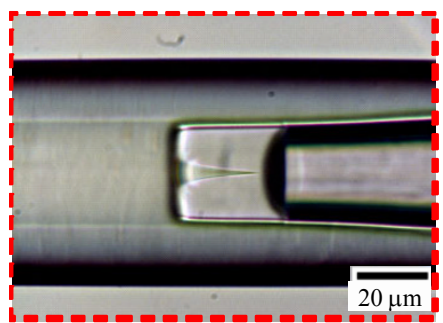

(c)

Fig. 10 Fibers with a protected internal glass tip were achieved by not applying the pre-etching step - the fiber tip, surrounded by a silica tube, is shown for different etching times: (a) $10 \mathrm{~min}$, (b) and (c) $90 \mathrm{~min}$.

In this case, an internal glass tip protected by a silica-jacket was formed. The sample in Fig. 10 was obtained from a $\mathrm{P}_{2} \mathrm{O}_{5}$-doped fiber and showed the progress of the structural shaping after an etching time $\left(t_{e}\right)$ of $10 \mathrm{~min}$ [Fig. 10(a)] and of $90 \mathrm{~min}$ [Figs. 10(b) and 10(c)]. Due to the higher etching rate of the central doped fiber layers, the etching occured mainly between the undoped central dip and the silica cladding. These structures could be considered as a fiber micro-reactor with an embedded optical tip sensor to analyze liquid or gaseous substances by using the evanescent field interactions at the interface between the micro tip and the surrounding mediums. It is also conceivable to implement such photonic devices in microfluidic systems.

Original fiber diameters of $125 \mu \mathrm{m}, 250 \mu \mathrm{m}$ and $500 \mu \mathrm{m}$ were tested for different formations of the internal tip structure in dependence on the etch processing. The graphs in Fig. 11 show the development of the inner fiber diameter, the cavity depth and the tip length depending on the starting diameter and on the etching time. The dimension of the fiber tips could be adjusted in a wide range and adapted to possible optical sensor applications.

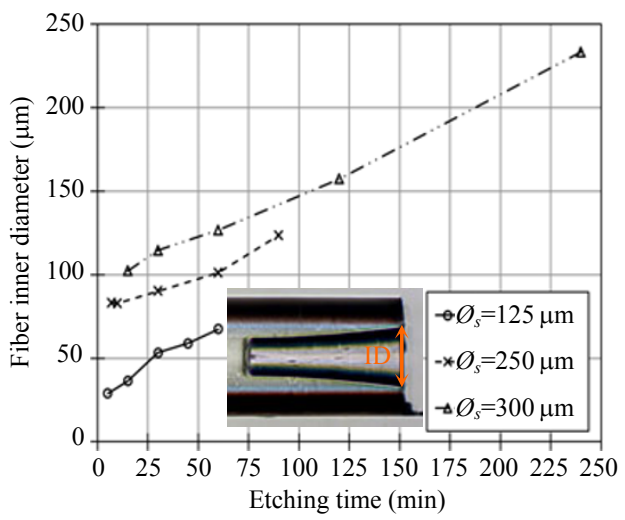

(a)

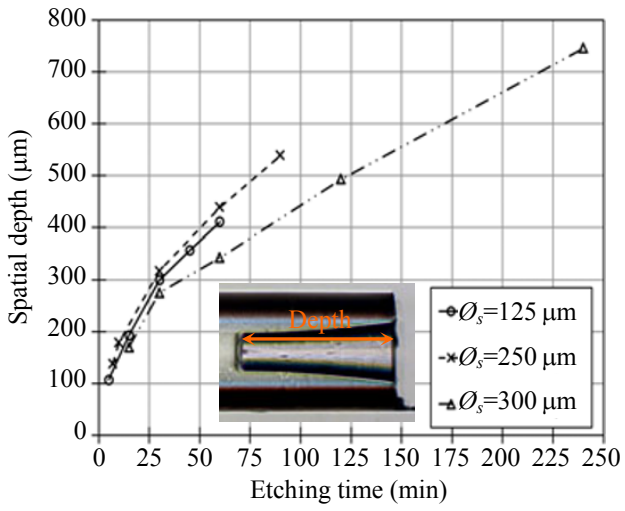

(b)

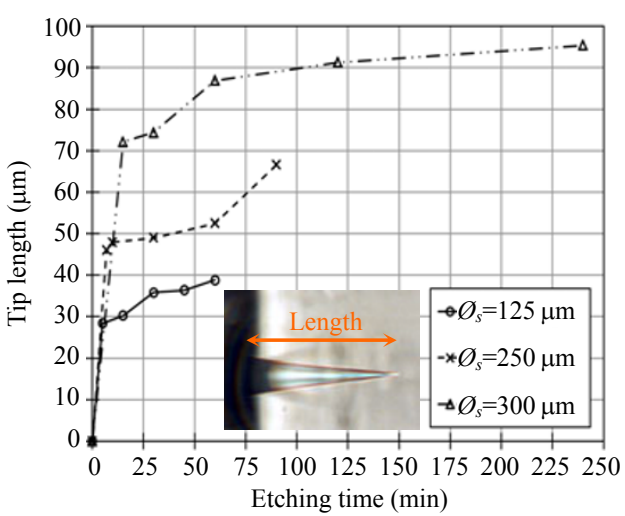

(c)

Fig. 11 Depending on the starting fiber diameter $\left(\varnothing_{s}\right)$ and on the etching time, the dimensions of the fiber, the cavity and the remaining tip can be varied. 


\section{Metallization of the glass fiber tips}

The tip probe of a near-field scanning optical microscope can be used in different operation modes with or without a metal coating. In the case of an apertureless operation mode, the metallization of the scanning tip is necessary for excitation of plasmon polariton waves. We have therefore investigated the coating of the exposed fiber tips with nanometer-sized silver layers $(20 \mathrm{~nm}-40 \mathrm{~nm}$ in thickness). Sputter deposition [Fig. 12(a)] as well as evaporation deposition [Fig. 12(b)] techniques have been used.

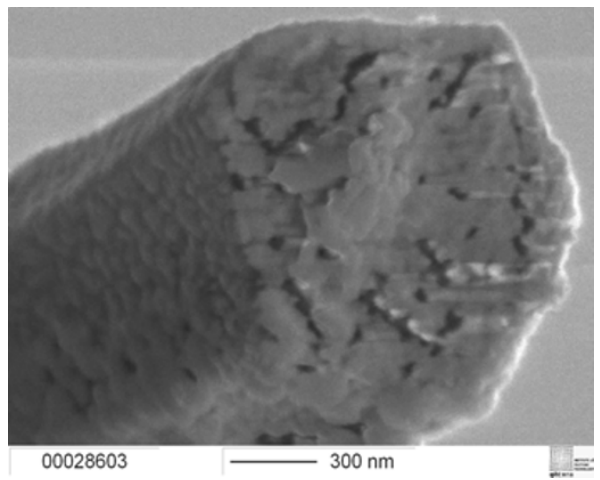

(a)

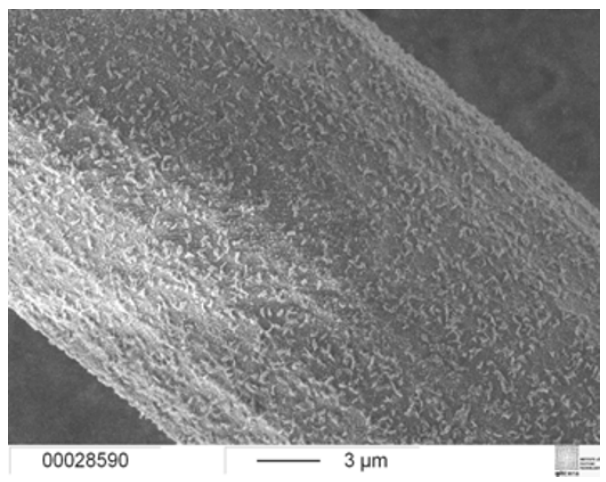

(b)

Fig. 12 Fiber tips were coated with nanometer-sized Ag-layers by sputtering (a) and evaporation deposition (b).

Depending on the deposition technique, different surface layer qualities were achieved. In order to obtain a droplet structure, the metal layers were subjected to a high temperature annealing process (Fig. 13). This structure is of advantage for localized excitation of plasmon resonances. The droplet formation occured at temperatures above $900{ }^{\circ} \mathrm{C}$ due to surface tension effects of the material in the melting phase. Silver has a low adhesion to glass and is therefore easily removable [Fig. 13(inset)]. Adhesion could be improved, e.g. by a titanium dioxide intermediate adhesive layer.

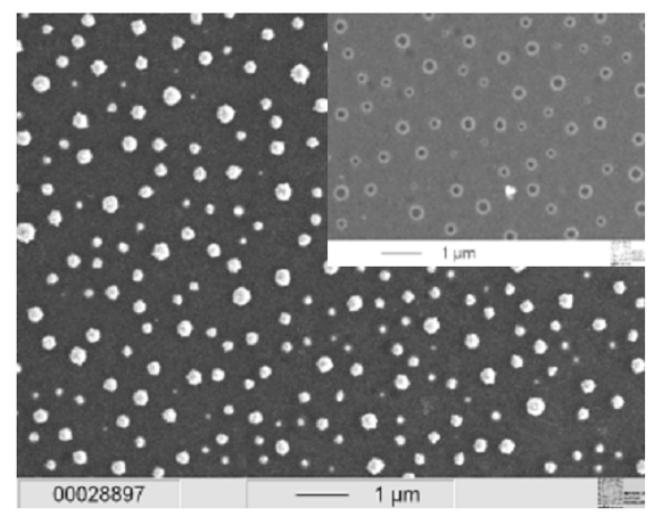

Fig. 13 Metal layers were converted into a droplet structure by a high temperature annealing treatment.

\section{Conclusions}

Studies on the preparation and characterization of gas phase etched optical fiber tips for nanoscopic sensor applications were performed. It was found that geometric shaping of glass tips strongly depended on the doping characteristics and on the etching procedures. Depending on the etching gas atmosphere, various tip geometry could be produced in a reproducible way. Different shaped fiber tips were prepared with pure silica and with phosphorus-doped glass fibers achieving tip radii down to less than $15 \mathrm{~nm}$. The fiber tip has been also built with a surrounding silica tube in order to create an open-path micro-reactor cell for chemical and refractive index sensing of liquid or gaseous analytes. Additionally, the fiber tips were metallized with a nanometer-sized silver-layer and structured by an annealing treatment. The use of specifically doped fibers could give new options for preparation of shaped fiber tips in nanoscopic imaging and photonic sensor applications. For the future work, the fiber structuring method proposed here, opens the possibility for manufacturing of micro- or nano-lensed fibers with an adjustable radius of lens 
curvature at the end of the glass tip structure to obtain a defined mode size for further photonic applications.

\section{Acknowledgment}

The authors would like to thank S. Unger and K. Hinze for preform preparation by MCVD as well A. Dellith, S. Hausschild and A. Scheffel for SEM investigations.

Open Access This article is distributed under the terms of the Creative Commons Attribution License which permits any use, distribution, and reproduction in any medium, provided the original author(s) and source are credited.

\section{References}

[1] Q. Zhong and D. Inniss, "Characterization of the lightguiding structure of optical fibers by atomic force microscopy," Journal of Lightwave Technology, vol. 12, no. 9, pp. 1517-1523, 1994.

[2] A. Lewis, M. Isaacson, A. Harootunian, and A. Murray, "Development of a $500 \AA$ spatial resolution light microscope: I. light is efficiently transmitted through $\lambda / 16$ diameter apertures," Ultramicroscopy, vol. 13, no. 3, pp. 227-231, 1984.

[3] D. W. Pohl, W. Denk, and M. Lanz, "Optical stethoscopy: image recording with resolution $\lambda / 20$," Applied Physics Letters, vol. 44, no. 7, pp. 651-653, 1984.

[4] F. Zenhausern, Y. Marthin, and H. K. Wickramasinghe, "Scanning interferometric apertureless microscopy: optical imaging at 10 angstrom resolution," Science, vol. 269, no. 5227, pp. 1083-1085, 1995.

[5] S. Mononobe and M. Ohtsu, "Fabrication of a pencil-shaped fiber probe for near-field optics by selective chemical etching," Journal of Lightwave Technology, vol. 14, no. 10, pp. 2231-2235, 1996.

[6] X. Zheng, D. Guo, Y. Shao, S. Jia, S. Xu, B. Zhao, et al., "Photochemical modification of an optical fiber tip with a silver nanoparticle film: a SERS chemical sensor," Langmuir, vol. 24, no. 8, pp. 4394-4398, 2008.

[7] G. Shambat, S. R. Kothapalli, A. Khurana, J. Provine, T. Sarmiento, K. Cheng, et al., "A photonic crystal cavity-optical fiber tip nanoparticle sensor for biomedical applications," Applied Physics Letters, vol. 100, no. 21 pp. 213702-1-213702-4, 2012.
[8] J. Zhang, X. Qiao, T. Guo, Y. Ma, R. Wang, Y. Weng, et al., "Highly-sensitive temperature sensor using a Hi-Bi fiber tip probe," IEEE Sensors Journal, vol. 12, no. 6, pp. 2077-2080, 2012.

[9] E. Cibula, S. Pevec, B. Lenardic, E. Pinet, and D. Donlagic, "Miniature all-glass robust pressure sensor," Optics Express, vol. 17, no 7, pp. 5098-5106, 2009.

[10] X. Chen, F. Shen, Z. Wang, Z. Huang, and A. Wang, "Micro-air-gap based intrinsic Fabry-Perot interferometric fiber-optic sensor," Applied Optics, vol. 45, no. 30, pp. 7760-7766, 2006.

[11] S. Pevec and D. Donlagic, "All-fiber, long-active-length Fabry-Perot strain sensor," Optics Express, vol. 19, no 16, pp. 15641-15651, 2011.

[12] S. Pevec and D. Donlagic, "All-fiber micro-machined Fabry-Perot strain sensor," in Proc. SPIE, Brussels, vol. 8426, pp. 84261D-1-84261D-7, 2012.

[13] D. Donlagic, "All-fiber micromachined microcell," Optics Letters, vol. 36, no. 16, pp. 3148-3150, 2011.

[14] S. Pevec, E. Cibula, B. Lenardic, and D. Donlagic, "Micromachining of optical fibers using the selective etching of doped silica glass," in Proc. SPIE, Brussels, vol. 8428, pp. 84280S-1-84280S-7, 2012.

[15] Y. Oshikane, T. Kataoka, M. Okuda, S. Hara, H. Inoue, and M. Nakano, "Observation of nanostructure by scanning near-field optical microscope with small sphere probe," Science and Technology of Advanced Materials, vol. 8, no. 3, pp. 181-185, 2007.

[16] P. Hoffmann, B. Dutoit, and R. P. Salathé, "Comparison of mechanically drawn and protection layer chemically etched optical fiber tips," Ultramicroscopy, vol. 61 , no. $1-4$, pp. 165-170, 1995.

[17] P. Lambelet, A. Sayah, M. Pfeffer, C. Philipona, and F. Marquis-Weible, "Chemically etched fiber tips for near-field optical microscopy: a process for smoother tips," Applied Optics, vol. 37, no. 31, pp. 7289-7292, 1998.

[18] Y. H. Chuang, K. G. Sun, C. J. Wang, J. Y. Huang, and C. L. Pan, "A simple chemical etching technique for reproducible fabrication of robust scanning near-field fiber probes," Review of Scientific Instruments, vol. 69, no. 2, pp. 437-439, 1998.

[19] H. Muramatsu, K. Homma, N. Chiba, N. Yamamoto, and A. Egawa, "Dynamic etching method for fabricating a variety of tip shapes in the optical fiber probe of a scanning near-field optical microscope," Journal of Microscopy, vol. 194, no. 2-3, pp. 383-387, 1999.

[20] L. Wong, T. Suratwala, M. D. Feit, P. E. Miller, and R. Steele, "The effect of $\mathrm{HF} / \mathrm{NH}_{4} \mathrm{~F}$ etching on the morphology of surface fractures on fused silica," Journal of Non-Crystalline Solids, vol. 355, no. 15, 
pp. 797-810, 2009.

[21] J. Bierlich, J. Kobelke, D. Brand, K. Kirsch, J. Dellith, and H. Bartelt, "Optical fiber tips for nanoscopic applications," in DGaO-Proc., Ilmenau, http://www.dgao-proceedings.de, ISSN: 1614-8436 - urn:nbn:de:0287-2011-P011-6, 2011.

[22] F. Dürr, G. Kulik, H. G. Limberger, R. P. Salathé, S. L. Semjonov, and E. M. Dianov, "Hydrogen loading and UV-irradiation induced etch rate changes in phosphorus-doped fibers," Optics Express, vol. 12, no. 23, pp. 5770-5776, 2004.
[23] Z. Yu, G. Collins, B. Harbison, I. Aggawal, D. Reicher, and J. McNeil, "Hermetic coatings on fluoride glass fibers using cylindrical magnetron reactive sputtering," Japanese Journal of Applied Physics, vol. 31, no. 12R, pp. 3969-3971, 1992.

[24] J. Bierlich, J. Kobelke, K. Schuster, J. Kupis, K. Kirsch, C. Aichele, et al., "Inorganic high temperature coatings for optical fibers by sputtering," in Proc. ICMAT 2009, Symposium on Optical Fiber Devices and Applications, Singapore, Jun 28-July 3, pp. 182-185, 2009. 